\title{
Publication Trends and Hot Spots in Chronic Postsurgical Pain (CPSP) Research: A I0-Year Bibliometric Analysis
}

\section{Qi Li iD* \\ Wanbing Dai* \\ Xuemei Chen \\ Diansan Su \\ Weifeng Yu \\ Xiyao Gu (D)}

Department of Anesthesiology, Renji Hospital, School of Medicine, Shanghai jiaotong University, Shanghai, 200I27,

People's Republic of China

*These authors contributed equally to this work
Correspondence: Xiyao Gu

Department of Anesthesiology, Renji

Hospital, School of Medicine, Shanghai

Jiaotong University, Shanghai, 200127,

People's Republic of China

Tel +86-2I-68383256

Email gxykevin@I26.com
Purpose: Aging populations and increasing quality of life requirements have attracted growing efforts to study chronic postsurgical pain (CPSP). However, a diverse range of factors are involved in CPSP development, which complicates efforts to predict and treat this disease. To advance research in this field, our study aimed to use bibliometric analysis to quantitatively and qualitatively evaluate CPSP research and predict research hot spots over the last 10 years.

Methods: Relevant publications between 2011 and 2020 were extracted from the Web of Science Core Collection database. CiteSpace software (v5.7.R2) and the Online Analysis Platform of Literature Metrology were used to analyze research attributes including countries and authors, keywords and co-occurrence, and burst detection to predict trends and hot spots. Results: A total of 2493 publications were collected with the number of annual publications showing nearly threefold increase over the past decade. Articles were the primary publication type with the United States as the leading country and the center of national collaboration. Johns Hopkins University provided the leading influence within the CPSP field. Postoperative pain, multimodal analgesia, quality of life, opioid, microglia, cesarean delivery, inguinal hernia, chronification, genetic polymorphism, and lidocaine were the top 10 clusters in co-occurrence cluster analysis. Moreover, burst detection was shown that epidural analgesia, nerve injury, total hip arthroplasty were the new hot spots within the CPSP field. Conclusion: Bibliometric mapping not only defined the overall structure of CPSP-related research but its collective information provides crucial assistance to direct ongoing research efforts. The prominent keywords including "risk factor" and "multimodal analgesia" indicate that CPSP prevention and new treatment methods remain hot spots. Nonetheless, the recognition that CPSP is complex and changeable, proposes comprehensive biopsychosocial approaches are needed, and these will be essential to improve CPSP interventions and outcomes.

Keywords: chronic postsurgical pain, bibliometric analysis, CiteSpace, co-citation analysis, burst detection with keywords

\section{Introduction}

The concept of chronic postsurgical pain (CPSP) was first introduced in 1999 and since that time its classification as a disease has been subject to ongoing refinement. The description for CPSP was expanded in $2001^{1,2}$ to involve "pain that occurs after surgical intervention and lasts at least 2 months" with the qualification that "other causes of pain must be ruled out, especially pain from a condition that occurred before the surgery". The definition was updated in 2014 to pain that occurs at the site of the incision or related areas of the surgery and persists a month longer than it 
takes for most injured tissues to fully heal. ${ }^{6}$ Consequently, the time of onset of CPSP has been primarily set between 3 and 6 months. $^{7}$ The definition of CPSP also differs depending on whether other causes of pain (such as disease recurrence after surgery or the presence of preexisting pain syndromes) are included under the CPSP rubric. $^{7,8}$

Depending on the type of surgery, the incidence of persistent pain after surgery varies from as low as 5\% up to $85 \% .^{3}$ For example, the average incidence of CPSP in amputations is $50 \%{ }^{4}$ compared with $10 \%$ following cesarean sections. ${ }^{5}$ Moreover, severe chronic postoperative pain has a negative impact on quality of life with this complication reported in an estimated $2 \%$ to $15 \%$ of the patients. ${ }^{6}$ Therefore, chronic postsurgical pain (CPSP) has become a top health priority and is included in the International Classification of Diseases (ICD-11). ${ }^{7}$ Since CPSP occurs at a specified point in time, it may be prevented and better controlled. However, many factors cause the occurrence and persistence of CPSP but only some of these are surgically related. Like non-surgical chronic pain, psychological and social factors also have a vital influence. ${ }^{8}$ All clinicians, and not just surgeons and anesthesiologists, should better understand how to recognize and manage CPSP, which may last for months or years after surgery. In daily clinical practice, the transition from acute pain after surgery to CPSP is often subtle and unpredictable And as with many other chronic diseases, early intervention may improve prognosis, so identifying at risk patients is crucial. Many studies have now investigated all aspects of CPSP ranging from prevention, diagnosis, and management along with the pathophysiological mechanisms involved. However, despite such efforts, the number of CPSP patients has not decreased. ${ }^{9}$

Bibliometric analysis is a new influence and evidence research and analysis framework. This approach uses bibliometric features to help researchers quantitatively identify detailed research trends, hot spots, and even joint citation networks with a certain field that collectively guide academic decisions. ${ }^{10}$ However, the application of quantitative methodology to the CPSP literature has been limited, let alone research on hot topics or trends that focus on analysis or prediction. In this study, we analyzed published CPSP studies worldwide between 2011 and 2020 to identify research trends and potential hot spots. We briefly discuss CPSP research and predict development trends in this field over the next few years.

\section{Materials and Methods Data Sources and Search Strategies}

We conducted a comprehensive search through the Web of Science Core Collection (WoSCC) database. The following search strategy was applied: [chronic postsurgical pain] OR [persistent postoperative pain], with the time frame set from 2011 to 2020 . We then screened and deleted some surgical-specific persistent postoperative pain, such as post-thoracotomy pain syndrome (PTPS). Publication type had no restriction and language was English. All literature retrieval and data downloads were completed in a single day, November 13, 2020, to reduce bias incurred by frequent database updates.

\section{Data Collection}

The manuscripts were screened and the titles, authors, countries, institutions, journals, and the total/average citation numbers were recorded and data then converted to txt format and imported into CiteSpace V5.7.R2 SE, 64 bit (Drexel University, Philadelphia, PA, USA) for further bibliometric analysis.

\section{Bibliometric Analysis}

All literature characteristics, including countries/regions, institutions, clustered networks of co-cited references and authors, and keywords with the strongest citation bursts were described. Online Bibliometric Analysis Platform and CiteSpace software were applied to visualize the bibliometric data. CiteSpace takes a collection of bibliographic records as input and creates a synthesized network based on a time series of networks derived from each year's publications to model the intellectual structure of the underlying domain. ${ }^{11}$ The size of a node in the network indicates the number of citations obtained by the related reference. Across the series of time slices, each node is represented by a series of citation tree rings. A colored ring is used to represent the structural properties of a node. The thickness of the ring represents the degree of centrality, which is a metric associated with the transformative potential of a scientific contribution. High centrality could be found in the hubs that are strongly connected nodes to other nodes and nodes in the middle of two classes of nodes. ${ }^{12}$ Burst detection is a computational technique for detecting sudden changes in events and other types of information. ${ }^{11}$ The details for calculation of centrality ${ }^{13}$ and burst detection ${ }^{14}$ have been previously described. 


\section{Results}

\section{Bibliometric Analysis of Publication Outputs}

A total of 2493 WoSCC publications met the inclusion criteria. Annual publication rates mostly demonstrated successive increases, although the number of papers appeared to drop in 2020, presumably related to COVID-19. Nonetheless, with close to a threefold rise from 2011 to 2018 (120 versus 346, respectively), this provides clear indications that CPSP research is gaining prominence. Indeed, of these, 1313 publications were published in the last 4 years, accounting for $52.6 \%$ of the total publications (Figure 1A).

Nine publication types concerning CPSP were identified in the past decade, with the prominent forms including articles, reviews and letters (Figure 1B). Original articles constituted the highest proportion (76.3\%) followed by reviews $(16.1 \%)$. The study locations involved 84 different countries/regions and among the total 2493 publications, the United States contributed the most (988), followed by Germany (198) and China (193) (Figure 1C). The manuscripts of these three countries accounted for more than half of all publications $(55.3 \%)$.

\section{Collaborating Countries and Institutions in CPSP Research}

A national/regional collaborating network analysis was conducted via CiteSpace. This analysis defined 84 nodes which clearly defined the United States as the center of national collaboration, also providing the most exchanges with other countries, followed by Germany and China (Figure 2A). Notably, this metric was strongly related to the number of US led publications. However, other countries/regions around the world also displayed a trend of prosperous exchange. African countries including Egypt and Saudi Arabia, together with South American countries such as Brazil and Colombia had close collaborations and showed regional clustering. Centrality assessment which indicates the importance and influence of nodes in the network, revealed that the United States had a much greater influence than any other country (centrality $=0.46$ ), followed by England (0.30) and Australia (0.28) (Table 1).

CiteSpace analysis of institutions identified 359 nodes, indicating 359 institutions were involved in the 2493 CPSP publications included in the analysis (Figure 2B). The research network presented a low-density map (density $=0.01$ ),

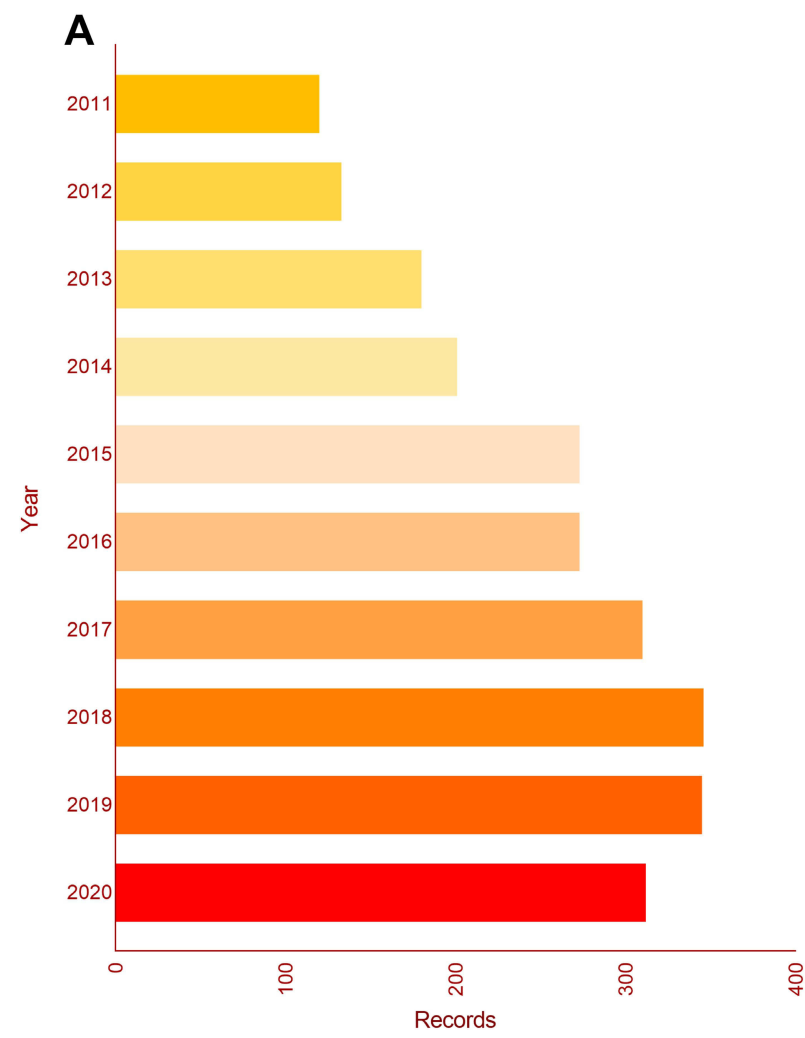

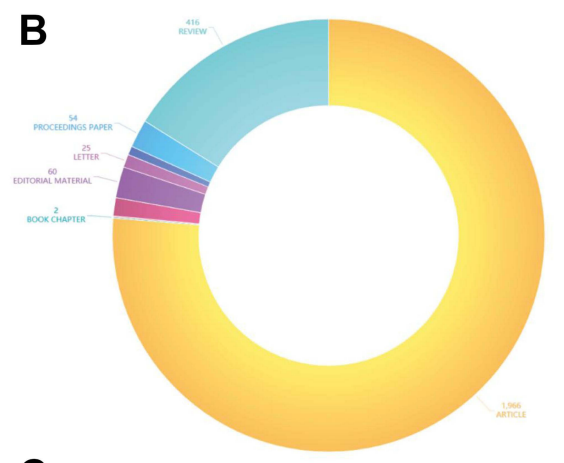

Article type

ARTICLE (76.3\%) - BOOK CHAPTER $(0.1 \%)$ CORRECTION (0.1\%) EARLY ACCESS $(1.3 \%$ EDITORIAL MATERIAL (2.3\%) - LETTER (1.0\%)

MEETING ABSTRACT (0.7\%) - PROCEEDINGS PAPER (2.1\%) - $\operatorname{REVIEW}(16.1 \%)$

C

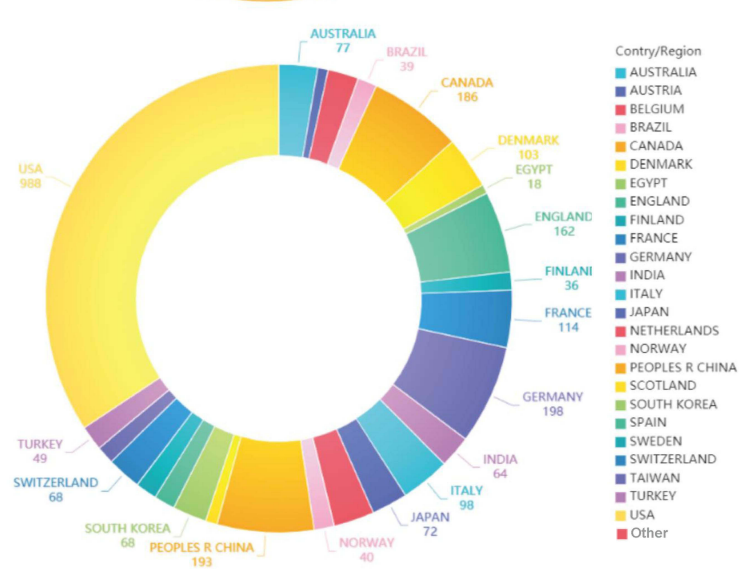

Figure I Bibliometric analysis of publication output. A total of 2493 publications were included from 20II to 2020. (A) The number of annual publications involving CPSP. (B) Article type distribution. (C) The distribution of publications by country/region. 

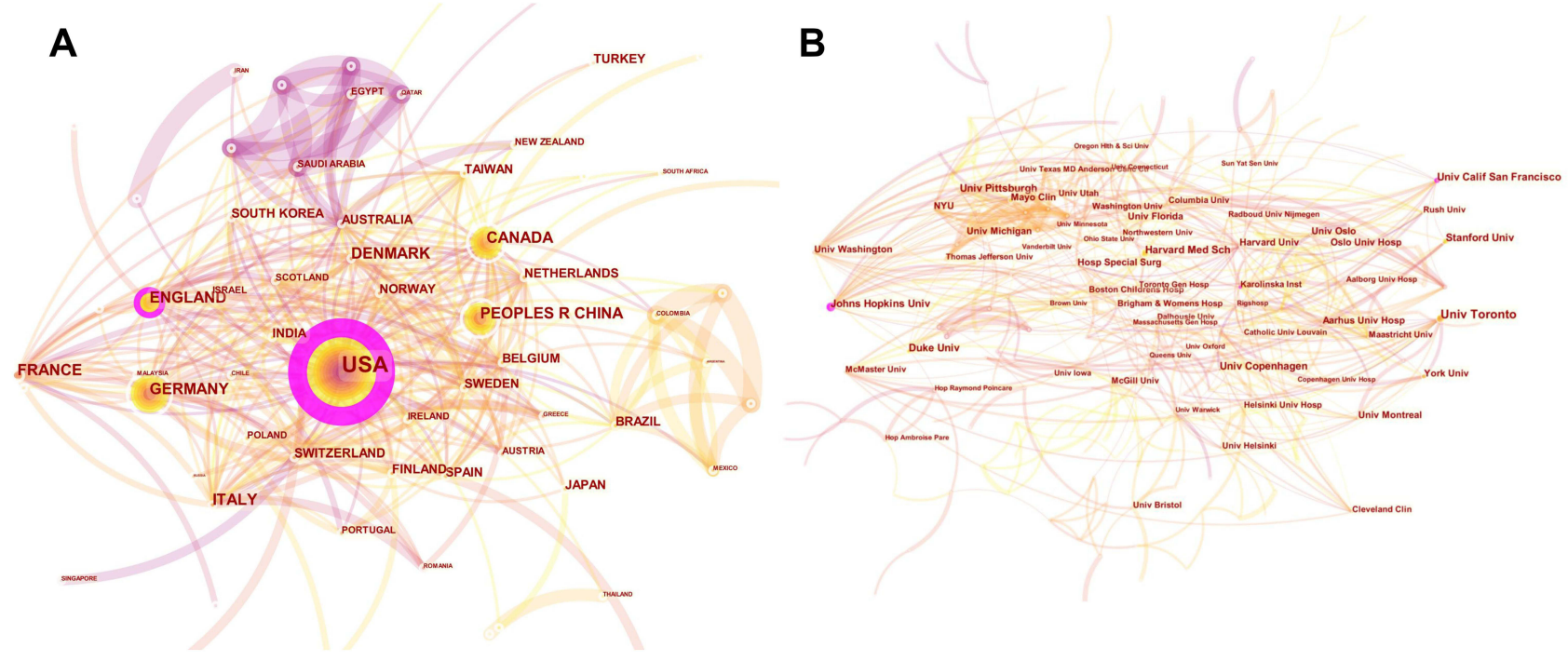

Figure 2 Collaborating countries, institutions and authors in CPSP research. The network map of collaborating countries/regions (A) and institutions (B) for CPSP research.

indicating that the research groups were relatively scattered in various institutions. The University of Toronto had the most frequent collaboration with other institutions while Johns Hopkins University has the leading influence (centrality $=0.31$ ), followed by the University of Washington (0.23). Harvard University and Karolinska Institute ranked third together (0.2, Table 2). Overall, these findings suggest more academic collaborations are needed.

\section{Analysis of Co-Cited Publications}

CiteSpace analysis identified 454 authors contributed to the 2493 CPSP research publications over the last decade. The cocited publications metric represents the scientific relevance of publications, ${ }^{10}$ with each node representing a cited article, and the size of each node is proportional to its total co-citation frequency (Figure 3A). The most cited publication with 96

Table I The Top 10 Countries/Regions for Centrality in CPSP Research

\begin{tabular}{|l|l|l|}
\hline Rank & Countries/Regions & Centrality \\
\hline 1 & The United States & 0.46 \\
2 & England & 0.30 \\
3 & Australia & 0.28 \\
4 & Canada & 0.27 \\
5 & Italy & 0.26 \\
6 & France & 0.25 \\
7 & Germany & 0.25 \\
8 & Sweden & 0.23 \\
9 & Denmark & 0.22 \\
10 & Belgium & 0.22 \\
\hline
\end{tabular}

citations was the review by Haroutiunian and colleagues published in PAIN in 2013. This article analyzed the occurrence of neuropathic pain induced by nerve injury after different types of surgery, which was attributed to the likelihood of surgical iatrogenic nerve injury. The authors identified large differences in the methodologies used in various studies, and concluded that future analysis requires more uniform methodology to evaluate persistent postoperative neuropathic pain. Clustering these articles shows that their main focus involved analgesics, hysterectomy and breast surgery (Figure 3B).

\section{Keyword Co-Occurrence Cluster Analysis of CPSP Hot Spots and Burst Detection with Keywords}

Co-occurrence cluster analysis using keywords from the 2011 to 2020 CPSP publications revealed usage of 541

Table 2 The Top 10 Institutions for Centrality in CPSP Research

\begin{tabular}{|l|l|l|}
\hline Rank & Institutions & Centrality \\
\hline 1 & Johns Hopkins University & 0.31 \\
2 & University of Washington & 0.23 \\
3 & Harvard University & 0.20 \\
4 & Karolinska Institutet & 0.20 \\
5 & Mayo Clinic & 0.18 \\
6 & University of Connecticut & 0.17 \\
7 & University of Toronto & 0.17 \\
8 & New York University & 0.17 \\
9 & University of Pittsburgh & 0.16 \\
10 & University of California, San Francisco & 0.16 \\
\hline
\end{tabular}


A

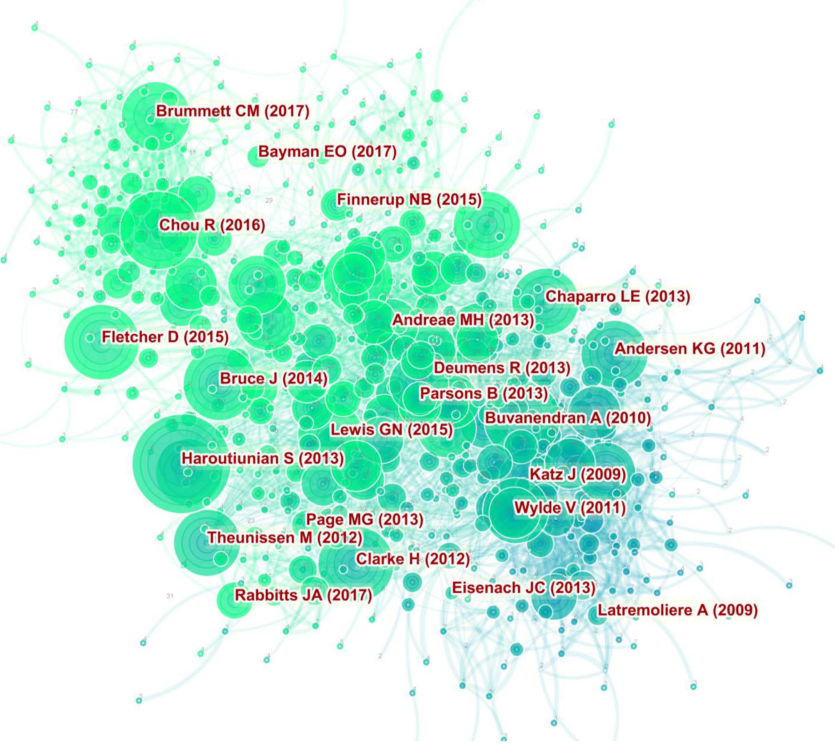

B

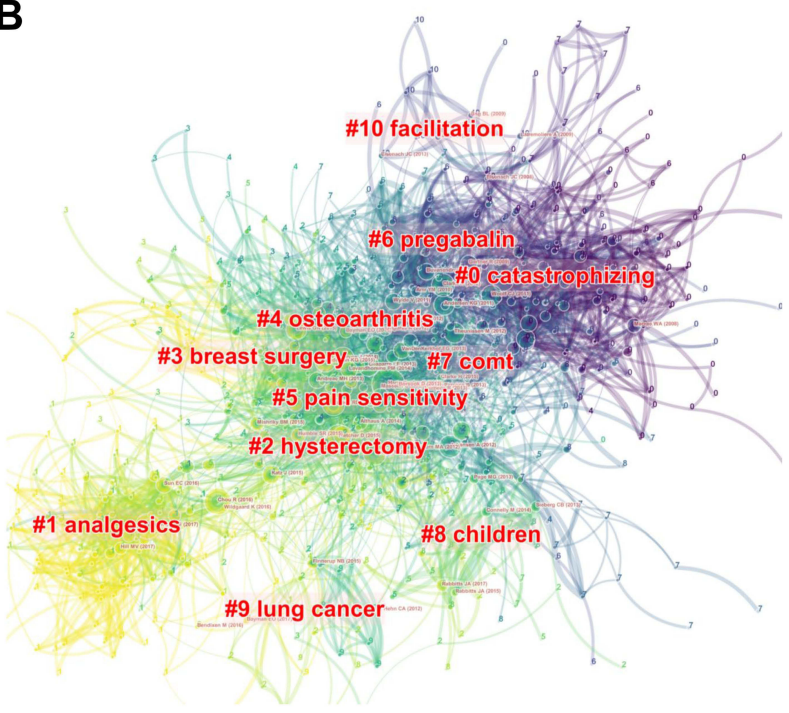

Figure 3 Co-cited authors and analysis of co-cited publications. The co-cited publication map (A) and clustered network map (B) of co-cited references of CPSP publications.

keywords. The position of the nodes represents the time when they occur, and the size represents the frequency of their occurrence, which reflects research hot spots. ${ }^{10}$ Unsurprisingly, the most frequent occurrence involved postoperative pain (cluster 0 ), while the other top 10 cluster labels were multimodal analgesia, quality of life, opioid, microglia, cesarean delivery, inguinal hernia, chronification, genetic polymorphism and lidocaine (Figure 4). Moreover, "risk factor" (463) was the most frequent keyword in co-occurrence analysis (Figure 5A).

Burst detection was performed to identify the emerging concepts that have drawn the attention of peer investigators.

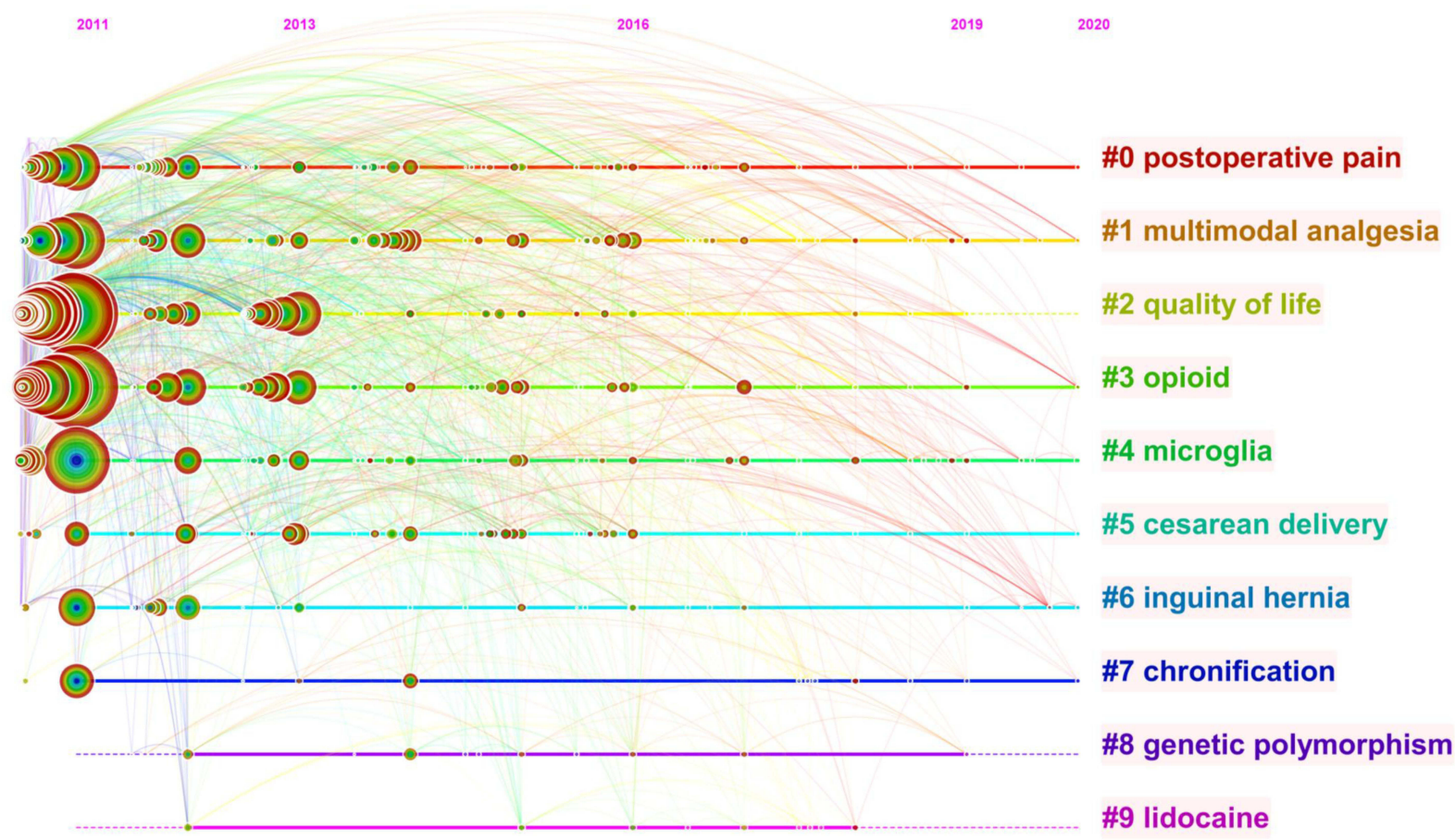

Figure 4 The timeline view of keyword clusters. 


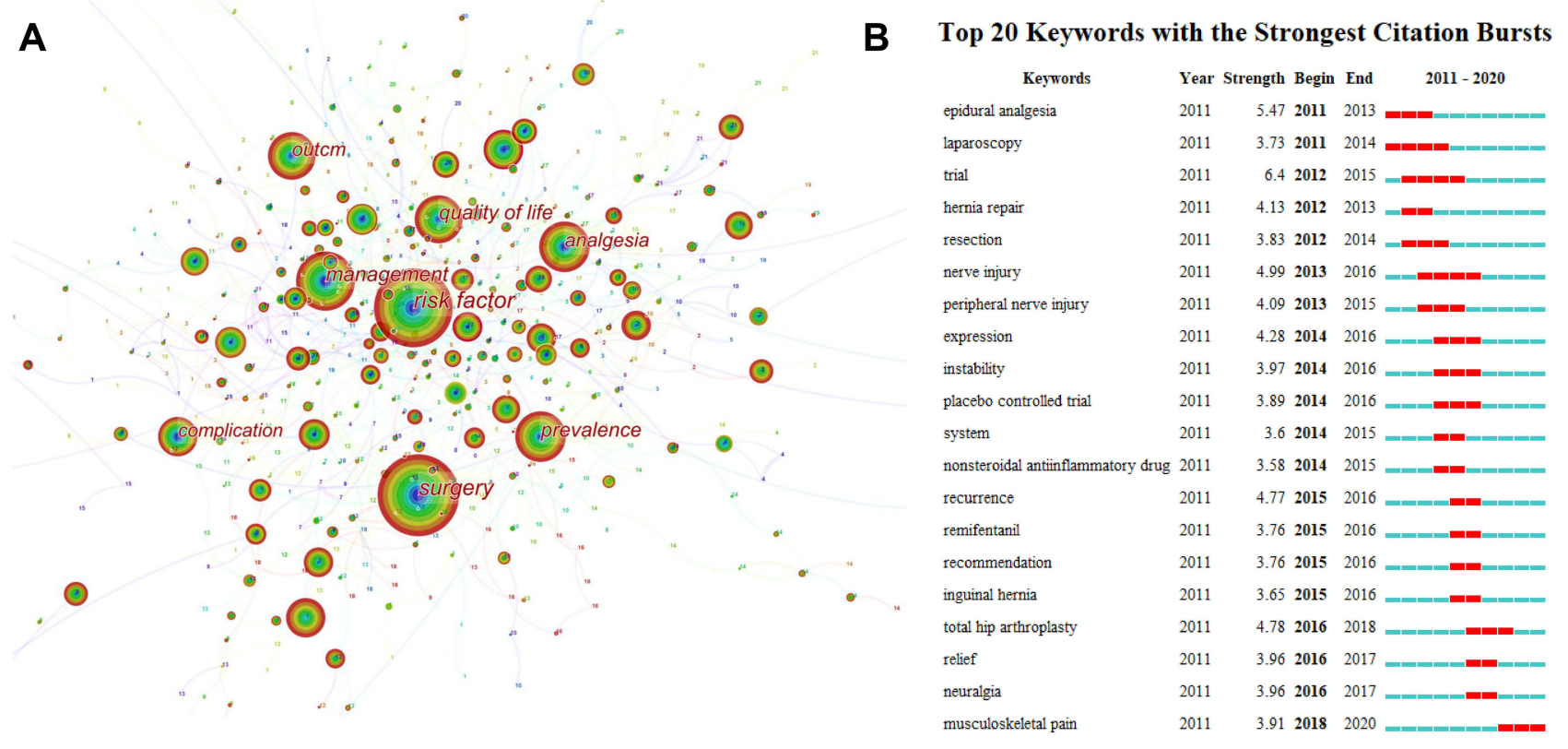

Figure 5 Keyword co-occurrence analysis of CPSP hot spots and burst detection with keywords. The network map of the co-occurrence keywords (A) and keywords with the strongest citation bursts (B) in original publications on CPSP research between $20 \mathrm{II}$ and 2020.

The blue line sliced by year refers to the timeline, and the time interval of a burst is marked as a red grid on the blue line, indicating the beginning and ending year and duration of a citation burst (Figure 5B). Over the past decade, trial ranked first with the highest burst strength (6.4), followed by epidural analgesia (5.47), nerve injury (4.99), total hip arthroplasty (4.78) and recurrence (4.77). Epidural analgesia and laparoscopy became the focus from 2011, followed by trial, hernia repair, resection, and nerve injury, with a duration of 2-4 years. Notably, recent attention involves musculoskeletal pain, indicating this is a current research hotspot.

\section{Discussion}

This study used bibliometrics methods to visually analyze a total of 2493 CPSP publications from 2011 to 2020, predominantly involving articles (76.1\%) and reviews (16.1\%). According to the results of qualitative and quantitative analysis conducted using the Online Bibliometric Analysis Platform and CiteSpace software, scientific research outputs involving CPSP has significantly increased over the past 10 years. The United States was the leading country for publications and the center of national collaboration with Johns Hopkins University providing the leading influence within the CPSP field. Postoperative pain, multimodal analgesia, quality of life, opioid, microglia, cesarean delivery, inguinal hernia, chronification, genetic polymorphism, and lidocaine were the top 10 clusters in co-occurrence cluster analysis. Moreover, burst detection showed that epidural analgesia, nerve injury, total hip arthroplasty were the new hot spots within the CPSP field.

With the exception of 2020, the number of CPSP related publications has increased year by year. In 2018, publication numbers even reached three times that of 2011, likely indicating that the increasing focus of quality of life issues has brought more attention to the topic of CPSP. Consistently, CPSP research was prominent in countries with higher economic development, with the United States first followed by Germany publishing the most CPSP research over the past decade. Indeed, excepting China, the top 10 contributing countries were all highly developed nations. Putting aside any potential racial differences like genetic susceptibility, this trend may be closely related to the expectations of high quality medical outcomes in these countries. With improvements in economies and technological developments, there is no doubt that CPSP will become an even stronger research focus in the future.

Our analysis of the highly cited articles and keywords revealed that the word "risk factor", ie, the prediction of CPSP, was the current hot spot in the CPSP field. The foremost critical factor leading to the development of CPSP involves neuropathic pain caused by nerve injury during surgery. ${ }^{15}$ Indeed as pointed out in the most cited review article involving CPSP by Haroutiunian et al 2013, persistent postoperative pain was most often reported as 
neuropathic pain. ${ }^{15}$ Moreover, it is considered that CPSP was closely related to the type of surgery ${ }^{16}$ and this notion is supported by data showing differing CPSP incidences. The reported rates of CPSP occurring in surgeries in the thoracic/breast area, bone and joint surgeries and surgeries on abdominal visceral structures were about $30-35 \%, 20$ and $10-14 \%$, respectively. ${ }^{15}$ The most influential publication detected involved a multicenter cohort study published by Montes and colleagues in Anesthesiology in 2015 also has a similar view. ${ }^{16}$ In addition, these authors grouped the risk models into six clinical predictors: surgical procedure, age, physical health, mental health, preoperative pain in the surgical field, and preoperative pain in another area. Indeed, pre-existing pain may lead to the development of persistent pain after surgery. The intensity of acute pain after breast surgery, thoracotomy, and inguinal hernia repair was also associated with the subsequent development of chronic pain. ${ }^{17,18}$ As for psychological factors, the theory on the development of CPSP had changed from a biomedical model to a biological, psychological, and social model. Anxiety, fear, past memories, social environment, expectations of work and physical activity, and other psychological issues were all thought to affect the development of CPSP. ${ }^{19}$ Taken together, this illustrates that the different CPSP risk factors operate interdependently. Moreover, due to the diverse and interactive nature of the factors involved in CPSP development, the prediction and prevention of CPSP had become complicated and cumbersome. Research on the pathogenesis of CPSP is also relatively scattered, and a systematic and recognized theory has not yet fully formed.

"Analgesia" also represents another key attraction for CPSP researchers based on the clustered network map of co-cited references and keyword co-occurrence cluster analysis. As a key part of surgery, analgesia is considered a key factor in the prevention of CPSP. Earlier studies had shown that the acute neuroplastic responses occurring with tissue damage could be prevented by active early analgesia. ${ }^{20}$ However, it was not clear whether techniques such as pre-emptive or preventive analgesia could effectively reduce the intensity or duration of postoperative pain. As single analgesia had various limitations, multimodal analgesia has become a research hot $\operatorname{spot}^{21}$ and as our results show, it is the second most frequently occurring keyword cluster. The eighth ranked article based on citations published by Clarke and colleagues in Anesthesia \& Analgesia in 2012 found that perioperative administration of gabapentin and pregabalin were effective in reducing the incidence of CPSP. ${ }^{22}$ During the perioperative period, the use of ketamine or gabapentin or pregabalin, COX inhibitors, steroids and other nerve block multimodal methods may also block central nervous system plasticity. $^{23,24}$ Therefore, optimizing perioperative pain management shows great potential for reducing the incidence of CPSP; however, fully conclusive evidence is still lacking, and further research is needed involving multimodal analgesia.

CPSP is increasingly regarded as a public health problem, not only because of the discomfort, pain, and disability it causes but also because past treatment approaches have served to aggravate the current opioid crisis. ${ }^{25}$ Indeed, the keyword "opioid" was highly attractive to researchers, ranking just after multimodal analgesia and quality of life (Figure 4). For a considerable time, opioid administration has been the major approach to CPSP management. However, postoperative opioid prescriptions vary widely, and are often overprescribed, even after minor surgery. In the United States, nearly 530 people die every week from opioid overdose. ${ }^{26}$ Patients undergoing surgery have an increased risk of long-term opioid use, and it remains particularly important to find ways to minimize this risk. ${ }^{27}$ Consequently, opioids have surged to become one of the hotspots of CPSP research over the past decade. This research is often dedicated to finding new therapeutic options to replace traditional opioid therapy. Since 2011, researchers had deepened their understanding of the neuropharmacology of sensory pathways and related structures (such as microglia) and established new targets. ${ }^{28}$ Novel opioids, adrenergic agonists, oxytocin, and cannabinoids were hot targets in recent years. ${ }^{29}$ The two modalities for which success has been achieved includes calcium channel blockers, for example, gabapentin and pregabalin, and agents that induce reuptake of serotonin and noradrenaline by monoamine transporters. ${ }^{21,30}$ Ziconotide is another targeted inhibitor of N-type calcium channels and although an effective analgesic, the narrow therapeutic window and requirement for intrathecal administration means it is rarely used clinically. ${ }^{31}$ Although these drugs were considered promising for effective treatment of CPSP, the clinical experience with gabapentin, serotonin, and norepinephrine reuptake inhibitors showed that these drugs were unlikely to successfully solve the complex problems caused by chronic postoperative pain. ${ }^{32}$ New methods including targeted toxins, gene-based methods such as protein synthesis blockade and transfection, and deep brain stimulation may therefore be useful. ${ }^{33}$ 
The current study has some limitations which must be acknowledged. First, the study design was limited to articles in the Web of Science Core Collection database and may therefore not include relevant articles only found in other sources such as Medline, the Cochrane library and Google Scholar. Second, relevant studies may have been missed if the search term "chronic postsurgical pain" or "persistent postoperative pain" was not included in the title, abstract, author keywords, keywords, or journal title. Third, this study may have missed some publications that did not include similar terms such as "chronic postoperative pain" or "persistent postural pain", as it is possible to retrieve other pain syndromes beyond the scope of CPSP. Finally, while citation rates drive the analysis metrics used in this study, citations are influenced by many factors and do not inherently represent the quality of the work involved. Nevertheless, we believe that this metric provides a valid representation of influence within this research field on a global scale. As the first bibliometric analysis of CPSP research, this study will help understand the development trends and research hotspots in this field.

\section{Conclusions}

Aging populations together increased access to surgical interventions are factors expected to drive the increasing incidence of CPSP. The annual related publications have nearly tripled in the past decade, with original articles being the primary type of publication. Research over the past 10 years has also been fruitful, establishing the foundation for CPSP research and defining new research directions. Our bibliometric mapping described the overall structure of scientific research for this field and provided collective information to assist other researchers. Prevention and new treatment methods of CPSP remain major focuses in the CPSP field. However, as CPSP is complex and changeable disease, a comprehensive biopsychosocial approach is needed for treatment, which is essential for developing new interventions and improving the prognosis of CPSP patients.

\section{Data Sharing Statement}

The raw data can be directly obtained from the Web of Science Core Collection (WoSCC) database.

\section{Acknowledgments}

This work was supported by grants from the National Natural Science Foundation of China (No. 81701092),
Shanghai Municipal Education Commission-Gaofeng Clinical Medicine Grant Support (20171916). The authors would like to express their gratitude to EditSprings (https://www.editsprings.com/) for the expert linguistic services provided.

\section{Disclosure}

The authors report no conflicts of interest in this work.

\section{References}

1. Macrae WA. Chronic pain after surgery. Br J Anaesth. 2001;87 (1):88-98. doi:10.1093/bja/87.1.88

2. Crombie HD. The beginnings of the New England surgical society. Arch Surg. 1998;133(4):352-353. doi:10.1001/archsurg.133.4.352

3. Kehlet H, Jensen TS, Woolf CJ. Persistent postsurgical pain: risk factors and prevention. Lancet. 2006;367(9522):1618-1625. doi:10.1016/S0140-6736(06)68700-X

4. Jung BF, Ahrendt GM, Oaklander AL, Dworkin RH. Neuropathic pain following breast cancer surgery: proposed classification and research update. Pain. 2003;104(1-2):1-13. doi:10.1016/S03043959(03)00241-0

5. Nikolajsen L, Sorensen HC, Jensen TS, Kehlet H. Chronic pain following Caesarean section. Acta Anaesthesiol Scand. 2004;48 (1):111-116. doi:10.1111/j.1399-6576.2004.00271.x

6. Fletcher D, Stamer UM, Pogatzki-Zahn E, et al. Chronic postsurgical pain in Europe: an observational study. Eur J Anaesthesiol. 2015;32 (10):725-734. doi:10.1097/EJA.0000000000000319

7. Schug SA, Lavand'homme P, Barke A, et al. The IASP classification of chronic pain for ICD-11: chronic postsurgical or posttraumatic pain. Pain. 2019;160(1):45-52. doi:10.1097/j.pain.00000 00000001413

8. Glare P, Aubrey KR, Myles PS. Transition from acute to chronic pain after surgery. Lancet. 2019;393(10180):1537-1546. doi:10.1016/ S0140-6736(19)30352-6

9. Weiser TG, Haynes AB, Molina G, et al. Size and distribution of the global volume of surgery in 2012. Bull World Health Organ. 2016;94 (3):201-209F. doi:10.2471/BLT.15.159293

10. Chen C, Song M. Visualizing a field of research: a methodology of systematic scientometric reviews. PLoS One. 2019;14(10):e0223994. doi:10.1371/journal.pone.0223994

11. Chen C. Science mapping: a systematic review of the literature. J Data Inf Sci. 2017;2(2):1-40. doi:10.1515/jdis-2017-0006

12. Chen $\mathrm{C}, \mathrm{Hu} \mathrm{Z}$, Liu S, Tseng H. Emerging trends in regenerative medicine: a scientometric analysis in CiteSpace. Expert Opin Biol Ther. 2012;12(5):593-608. doi:10.1517/14712598.2012.674507

13. Brandes U. A faster algorithm for betweenness centrality. $J$ Math Sociol. 2001;25(2):163-177. doi:10.1080/0022250X.2001.9990249

14. Kleinberg J. Bursty and hierarchical structure in streams. Data Min Knowl Discov. 2003;7:373-397. doi:10.1023/A:1024940629314

15. Haroutiunian S, Nikolajsen L, Finnerup NB, Jensen TS. The neuropathic component in persistent postsurgical pain: a systematic literature review. Pain. 2013;154(1):95-102. doi:10.1016/j. pain.2012.09.010

16. Montes A, Roca G, Sabate S, et al. Genetic and clinical factors associated with chronic postsurgical pain after hernia repair, hysterectomy, and thoracotomy: a two-year multicenter cohort study. Anesthesiology. 2015;122(5):1123-1141. doi:10.1097/ALN.0000000 000000611

17. Manangi M, Shivashankar S, Vijayakumar A. Chronic pain after inguinal hernia repair. Int Sch Res Notices. 2014;2014:839681. doi:10.1155/2014/839681 
18. Hetmann F, Kongsgaard UE, Sandvik L, Schou-Bredal I. Postthoracotomy pain syndrome and sensory disturbances following thoracotomy at 6- and 12-month follow-ups. $J$ Pain Res. 2017;10:663-668. doi:10.2147/JPR.S126639

19. Schug SA, Bruce J. Risk stratification for the development of chronic postsurgical pain. Pain Rep. 2017;2(6):e627. doi:10.1097/ PR9.0000000000000627

20. Coderre TJ, Katz J. Peripheral and central hyperexcitability: differential signs and symptoms in persistent pain. Behav Brain Sci. 1997;20(3):404-419. doi:10.1017/S0140525X97251484

21. Wick EC, Grant MC, Wu CL. Postoperative multimodal analgesia pain management with nonopioid analgesics and techniques: a review. JAMA Surg. 2017;152(7):691-697. doi:10.1001/jamasurg.20 17.0898

22. Clarke H, Bonin RP, Orser BA, Englesakis M, Wijeysundera DN, Katz J. The prevention of chronic postsurgical pain using gabapentin and pregabalin: a combined systematic review and meta-analysis. Anesth Analg. 2012;115(2):428-442. doi:10.1213/ANE.0b01 $3 \mathrm{e} 318249 \mathrm{~d} 36 \mathrm{e}$

23. Horn A, Kaneshiro K, Tsui BCH. Preemptive and preventive pain psychoeducation and its potential application as a multimodal perioperative pain control option: a systematic review. Anesth Analg. 2020;130(3):559-573. doi:10.1213/ANE.0000000000004319

24. Clarke H, Poon M, Weinrib A, Katznelson R, Wentlandt K, Katz J. Preventive analgesia and novel strategies for the prevention of chronic post-surgical pain. Drugs. 2015;75(4):339-351. doi:10.1007/s40265-015-0365-2

25. Baker DW. History of the joint commission's pain standards: lessons for today's prescription opioid epidemic. JAMA. 2017;317 (11):1117-1118. doi:10.1001/jama.2017.0935
26. Brummett CM, Waljee JF, Goesling J, et al. New persistent opioid use after minor and major surgical procedures in US adults. JAMA Surg. 2017;152(6):e170504. doi:10.1001/jamasurg.2017.0504

27. Hah JM, Bateman BT, Ratliff J, Curtin C, Sun E. Chronic opioid use after surgery: implications for perioperative management in the face of the opioid epidemic. Anesth Analg. 2017;125(5):1733-1740. doi:10.1213/ANE.0000000000002458

28. Li T, Liu T, Chen X, et al. Microglia induce the transformation of A1/ A2 reactive astrocytes via the CXCR7/PI3K/Akt pathway in chronic post-surgical pain. $J$ Neuroinflammation. 2020;17(1):211. doi:10.1186/s12974-020-01891-5

29. Yaksh TL, Woller SA, Ramachandran R, Sorkin LS. The search for novel analgesics: targets and mechanisms. F1000Prime Rep. 2015;7:56. doi:10.12703/P7-56

30. Marks DM, Shah MJ, Patkar AA, Masand PS, Park GY, Pae CU. Serotonin-norepinephrine reuptake inhibitors for pain control: premise and promise. Curr Neuropharmacol. 2009;7(4):331-336. doi:10.2174/157015909790031201

31. Patel R, Montagut-Bordas C, Dickenson AH. Calcium channel modulation as a target in chronic pain control. Br J Pharmacol. 2018;175 (12):2173-2184. doi:10.1111/bph.13789

32. Volkow ND, Collins FS. The role of science in the opioid crisis. N Engl J Med. 2017;377(18):1798. doi:10.1056/NEJMsr1706626

33. Barragan-Iglesias $\mathrm{P}$, Lou TF, Bhat VD, et al. Inhibition of Poly(A)-binding protein with a synthetic RNA mimic reduces pain sensitization in mice. Nat Commun. 2018;9(1):10. doi:10.1038/ s41467-017-02449-5
Journal of Pain Research

\section{Publish your work in this journal}

The Journal of Pain Research is an international, peer reviewed, open access, online journal that welcomes laboratory and clinical findings in the fields of pain research and the prevention and management of pain. Original research, reviews, symposium reports, hypothesis formation and commentaries are all considered for publication. The manuscript management system is completely online and includes a very quick and fair peer-review system, which is all easy to use. Visit http:// www.dovepress.com/testimonials.php to read real quotes from published authors. 